\title{
Optimal First-Line and Maintenance Treatments for Advanced-Stage Nonsquamous Non-Small Cell Lung Cancer
}

\author{
Ryan D. Gentzler, MD, MS, and Jyoti D. Patel, MD
}

\begin{abstract}
During the past 10 years, the treatment of advanced-stage nonsmall cell lung cancer (NSCLC) has become increasingly complex, and debate continues regarding the optimal chemotherapeutic agents and duration of treatment. The addition of bevacizumab to platinum doublet chemotherapy, the use of pemetrexed for nonsquamous histology, and the introduction of maintenance chemotherapy are strategies that have been shown to improve overall survival beyond 12 months. Many acceptable treatment options are recommended in the NCCN Clinical Practice Guidelines in Oncology for NSCLC. This article discusses the first-line treatment of NSCLC with no identifiable mutations with FDA-approved targeted therapies for patients treated outside a clinical trial, particularly focusing on difficult clinical decisions, such as when the use of bevacizumab is appropriate, choosing a platinum partner, and treatment of patients with an ECOG performance status of 2. Data are summarized from several recent maintenance clinical trials, such as PARAMOUNT, AVAPERL, and PointBreak, and the implications these trials have on practical decisions oncologists must make when choosing an optimal treatment strategy for patients with advanced NSCLC are discussed. ( $\mathrm{N}$ Natl Compr Canc Netw 2014;12:889-897)
\end{abstract}

During the past 10 years, the treatment of advancedstage non-small cell lung cancer (NSCLC) has become increasingly complex. From the use of newer chemotherapeutics in platinum-based doublets to the introduction of targeted agents and maintenance chemotherapy, the decisions facing oncologists when treating patients with newly diagnosed NSCLC are more nuanced than in the past. Despite large clinical trials that have contributed significant understanding to the treatment of lung cancer, debate continues regarding the optimal chemotherapeutic agents and duration of treatment. Unfortunately, despite investment of considerable patient and research resources, fewer than $5 \%$ of patients who present with metastatic disease are alive 5 years after diagnosis. ${ }^{1}$

It is well-known that patients with NSCLC are increasingly more heterogeneous than previously thought. The identification of specific genotypic abnormalities has allowed for the development and use of targeted therapies in the first-line setting, such as erlotinib and crizotinib for epidermal growth factor receptor (EGFR)-mutated and anaplastic lymphoma kinase (ALK)-rearranged NSCLC, respectively. This article focuses on the first-line treatment of NSCLC with no identifiable mutations with FDA-approved targeted therapies for patients treated outside a clinical trial. In this setting, the NCCN Clinical Practice Guidelines in Oncology (NCCN Guidelines) recommend several options for first-line therapy (to view the most recent version of these guidelines, visit NCCN.org). ${ }^{2}$ Histology has also become particularly relevant for treatment decisions, and patients with squamous histology NSCLC are not eligible for treatment with pemetrexed or bevacizumab, 2 key drugs that will be discussed in detail. For this reason, the discussion will primarily focus on patients who have nonsquamous histology, absence of EGFR or ALK mutations, and advancedstage IIIB or IV NSCLC that is not amenable to surgery 
or concurrent chemotherapy and radiation. The truly optimal management of this disease is prevention through smoking cessation efforts, tobacco control, and implementation of screening programs to detect lung cancer at earlier stages.

\section{Background}

\section{Platinum-Based Doublets}

In the 1990s and early 2000s, a large meta-analysis and several subsequent clinical trials showed that chemotherapy prolonged survival and was associated with improved quality of life for patients with advanced NSCLC. ${ }^{3-5}$ Around the same time, several new chemotherapeutics became available for the treatment of NSCLC. The large phase III ECOG 1594 trial randomized 1155 patients with advanced NSCLC with a performance status (PS) of 0 to 2 to a reference regimen of cisplatin and paclitaxel versus 3 other platinum-based doublets using the new "thirdgeneration" agents: cisplatin and gemcitabine; cisplatin and docetaxel; and carboplatin and paclitaxel. ${ }^{6}$ The median overall survival (OS) was 7.9 months for all patients (95\% CI, 7.3-8.5 months), and no statistically significant differences in survival were seen among the 4 regimens. Patients receiving carboplatin and paclitaxel had a lower incidence of grade 3 and 4 nausea, vomiting, and febrile neutropenia. From this point on, carboplatin and paclitaxel became the most commonly used regimen in the United States for patients with advanced NSCLC, with the other 3 regimens remaining acceptable alternatives.

Many studies, including ECOG 1594, have used cisplatin or carboplatin as the platinum base for induction chemotherapy. A meta-analysis comparing outcomes for patients receiving cisplatin versus carboplatin who were treated on 8 different clinical trials found no significant difference in OS (hazard ratio [HR], 1.050; 95\% CI, 0.907-1.216; P=.515). ${ }^{?}$ Subgroup analysis of 5 trials that included "newer agents" at the time found a small improvement in OS for patients receiving cisplatin (HR, 1.106; 95\% CI, 1.005-1.218; $P=.039$ ). Despite these findings, routine use of cisplatin over carboplatin in palliative chemotherapy regimens should be cautioned because cisplatin provides only several weeks of OS benefit and it has a potential risk for increased toxicity, especially in elderly patients or those with comorbidities.

\section{Bevacizumab}

Bevacizumab is a monoclonal antibody directed at vascular endothelial growth factor (VEGF), which has been shown to be overexpressed in NSCLC and implicated in tumor angiogenesis. ${ }^{8}$ An early phase II trial randomized patients with NSCLC to carboplatin and paclitaxel plus bevacizumab versus carboplatin and paclitaxel alone. ${ }^{9}$ The results showed an improved response rate and OS for those receiving bevacizumab. Perhaps the most important finding was that 4 of 13 patients with squamous histology receiving bevacizumab had a life-threatening pulmonary hemorrhage, representing a much higher rate than patients with adenocarcinoma histology. The follow-up phase III ECOG 4599 study excluded patients with squamous histology. ${ }^{10}$ This study also excluded patients with brain metastases; bleeding diatheses or coagulopathy; or the use of anticoagulants, aspirin, or nonsteroidal anti-inflammatory agents because of the risk of bleeding or thrombosis associated with bevacizumab. Eligible patients with stage IIIB or IV nonsquamous NSCLC (NS-NSCLC) were treated with carboplatin plus paclitaxel with or without bevacizumab every 3 weeks for 6 cycles. Those in the bevacizumab arm who did not have disease progression continued on bevacizumab at $15 \mathrm{mg} / \mathrm{kg} \mathrm{ev}$ ery 3 weeks until disease progression or unacceptable toxicity. Patients who received bevacizumab had an improvement in median progression-free survival (PFS) and OS of 2 months (PFS, 6.2 vs 4.5 months; HR, 0.66; $P<.001$, and OS, 12.3 vs 10.3 months; HR, $0.79 ; P=.003)$, marking the first time a clinical trial improved median OS beyond 1 year for patients with metastatic NSCLC.

AVAiL, a second large, double-blind, phase III study randomized patients to 1 of 3 arms, 2 of which contained bevacizumab at different doses (7.5 and $15.0 \mathrm{mg} / \mathrm{kg}$ ) that was added to a chemotherapy regimen of cisplatin and gemcitabine. ${ }^{11}$ The study was designed with a primary end point of PFS and enrolled approximately 330 patients in each of the 3 arms. The trial met the primary end point of improved PFS for both bevacizumab arms when compared with the placebo arm. Median OS for all patients was more than 13 months, with no statistically significant difference between the bevacizumab and control arms. ${ }^{12}$

Although some may argue that the results of AVAiL contradict those of ECOG 4599 and ques- 
Optimal Treatment Strategies for Advanced NSCLC

tion the true OS benefit of the addition of bevacizumab to induction chemotherapy, several possible factors could explain the difference between these trials. The patient population in AVAiL had a higher proportion of favorable prognostic factors, such as earlier stage and never-smoker status. More patients in this study also received second-line chemotherapy $(62 \%)$ and were treated with erlotinib and pemetrexed, 2 newer agents that had become available after ECOG 4599.

Several meta-analyses of trials using bevacizumab have been conducted with conflicting results. ${ }^{13-15}$ The most recent meta-analysis evaluating 4 phase II and III studies concluded that bevacizumab was associated with an improvement in OS (HR, 0.72; 95\% CI, 0.66-0.79; $P<.001)$ and PFS (HR, 0.90; 95\% CI, $0.81-0.99 ; P=.03) .{ }^{15}$ This analysis validates the clinically significant improvement patients experience with the addition of bevacizumab. The chemotherapy to which bevacizumab is added may also impact the relative improvement it has over chemotherapy alone. This could possibly account for the lack of OS benefit seen in AVAiL, which used gemcitabine instead of paclitaxel. No randomized phase III trials have evaluated the effect of adding bevacizumab to pemetrexed-based chemotherapy. Many subsequent trials discussed herein have included bevacizumab in both arms, which could confound whether adding bevacizumab to pemetrexed-based chemotherapy provides additional benefit.

\section{Pemetrexed}

After bevacizumab, pemetrexed was the next agent to dramatically alter the standard approach to first-line treatment of NSCLC. Pemetrexed initially became available for the second-line treatment of NSCLC after a randomized phase III trial showed equivalent efficacy and an improved toxicity profile when compared with docetaxel. ${ }^{16}$ Subsequent first-line trials using pemetrexed have shown that response is dependent on histology, and consequently, the accurate assessment of histologic subtype within NSCLC has become increasingly important. The landmark trial by Scagliotti et $\mathrm{al}^{17}$ was the first to show that pemetrexed resulted in a survival advantage for NS-NSCLC. In this phase III noninferiority study, combination cisplatin and gemcitabine was compared with cisplatin and pemetrexed for patients with all histologic subtypes of advanced NSCLC, but its design included a preplanned subset analysis based on histology. The results showed no statistical difference between the arms in the entire population, but there was a significantly improved OS in the pemetrexed arm when compared with gemcitabine for patients with adenocarcinoma and large cell carcinoma histologies (12.6 vs 10.9 months and 10.4 vs 6.7 months, respectively). Patients with squamous histology had improved survival with gemcitabine (10.8 vs 9.4 months). These subgroup analyses each reached statistical significance. Based on these data, pemetrexed in combination with a platinum agent was approved by the FDA for the front-line treatment of NS-NSCLC.

\section{Maintenance}

Although this review focuses on the optimal firstline therapy for advanced NSCLC, maintenance considerations cannot be completely separate from those regarding induction chemotherapy, and the findings of several key maintenance trials influence decision-making about induction chemotherapy options. Importantly, however, a significant portion of patients, from $33 \%$ to $59 \%$ in most series, ${ }^{10,11,18-21}$ are not eligible for maintenance therapy because of the occurrence of progressive disease, death, or toxicity during induction chemotherapy. Therefore, selection of induction regimens should not be based primarily on prospective choices of maintenance therapy. Two maintenance strategies have been shown to be effective. With switch maintenance, 4 to 6 cycles of a platinum doublet is immediately followed by a new drug in patients without progressive disease. Some investigators think of this as early second-line therapy. Alternatively, with continuation maintenance, one or more drugs used in the induction regimen is continued after 4 to 6 cycles until progression. The first trial to suggest improved efficacy with a continuation maintenance strategy was a phase II trial of carboplatin, pemetrexed, and bevacizumab followed by pemetrexed and bevacizumab maintenance. ${ }^{22}$ This study showed long median PFS and OS of 7.8 and 14.1 months, respectively.

Subsequently, 3 large phase III trials, PARAMOUNT, PointBreak, and AVAPERL, have all evaluated the role of pemetrexed and/or bevacizumab in induction and/or maintenance in different ways. ${ }^{18,19,23}$ All 3 studies enrolled patients with advanced stage IIIB or IV NS-NSCLC, and patients were continued on maintenance therapy only if they did not experience disease progression after induction chemotherapy. 
In PARAMOUNT, patients were treated with 4 cycles of induction therapy with cisplatin and pemetrexed and were then randomized 2:1 to pemetrexed versus placebo, with a primary end point of PFS. The study met the primary end point goal with an improvement in PFS from 2.8 to 4.1 months for patients receiving pemetrexed maintenance ( $\mathrm{HR}$, $0.62 ; P<.0001) .{ }^{23}$ The updated results showed an improvement in OS of 3 months for those receiving maintenance pemetrexed (16.9 vs 14.0 months; HR, 078; $P=.0191) .{ }^{21}$ Other studies have demonstrated pemetrexed efficacy in the setting of secondline therapy ${ }^{16}$ or when used in a switch-maintenance strategy. ${ }^{24}$ PARAMOUNT provides further evidence that pemetrexed has activity in the first-line setting and leads to further improvement in OS when continued as a maintenance strategy.

PointBreak was the phase III follow-up study based on promising results of an earlier phase II study that showed safety and tolerability of a regimen using carboplatin, pemetrexed, and bevacizumab followed by maintenance pemetrexed and bevacizumab. The preceding phase II study was the first to evaluate the use of 2 agents in the maintenance portion of therapy and the first to combine pemetrexed and bevacizumab during induction. ${ }^{22}$ PointBreak randomized patients to carboplatin, pemetrexed, and bevacizumab versus carboplatin, paclitaxel, and bevacizumab followed by bevacizumab, which was the regimen used in ECOG 4599 and was believed to be the standard of care for bevacizumab-eligible patients. This was a randomized open-label phase III study with a primary end point of OS. Although a small and statistically significant improvement in PFS was seen in the group receiving the PointBreak regimen (6.0 vs 5.6 months; HR, 0.83; $P=.012)$, no improvement was seen in OS (12.6 vs 13.4 months; HR, 1.00; $P=.949) .{ }^{19}$ Similar to many other maintenance studies, only 598 of all 939 patients on study (63\%) received maintenance therapy, mostly attributable to progressive disease or death. Exploratory analysis of this group of patients showed an improved OS (17.7 vs 15.7 months) for those receiving the PointBreak regimen. Patients treated with the ECOG 4599 regimen had statistically significant higher rates of grade 3 and 4 neutropenia, febrile neutropenia, and peripheral neuropathy. The group receiving the PointBreak regimen had significantly higher grade 3 and 4 anemia, thrombocytopenia, and fatigue, likely explained by prolonged use of a cytotoxic agent in the maintenance phase. Alopecia was much lower, although not completely absent, in the PointBreak group ( $7 \%$ vs $37 \%$ ). The PointBreak study shows that the 2 regimens have equivalent efficacy, but the toxicity profiles differ greatly.

AVAPERL is a third maintenance study that used a PointBreak-like regimen of cisplatin, pemetrexed, and bevacizumab induction followed by pemetrexed and bevacizumab maintenance as the experimental $\mathrm{arm}$. The control arm used the same induction with only bevacizumab maintenance. ${ }^{25}$ The primary end point was PFS. Two-thirds of those enrolled received maintenance. Those receiving the PointBreak-like regimen showed a statistically significant improvement in median PFS (10.2 vs 6.6 months; HR, 0.50; $P<.001) .{ }^{18}$ Updated OS results were presented at the 2013 ASCO Annual Meeting. ${ }^{28}$ Although prolonged OS was seen in the combination maintenance arm, the study was underpowered to reach statistical significance ( 19.8 vs 15.9 months; HR, $0.88 ; P=.32$ ).

The data from the PARAMOUNT, PointBreak, and AVAPERL maintenance trials are clinically significant and practice-changing in several ways. First, all of the studies remind that approximately one-third of patients will have progressive disease or death during the first 3 months of induction chemotherapy and will be ineligible for maintenance. For those with disease response or stabilization of disease after induction chemotherapy, maintenance therapy with cytotoxic chemotherapy or bevacizumab, or the combination of both, provides a significant benefit. Other studies using different agents or strategies have also supported this concept. $20,24,26$ Whether additional benefit is derived from continuing bevacizumab when using pemetrexed maintenance remains unclear. Neither PointBreak nor AVAPERL is able to answer this question, because these trials continued bevacizumab in the maintenance phase in both arms.

Whether continuation of pemetrexed alone is different from continuation of both pemetrexed and bevacizumab is currently unclear. When choosing a pemetrexed-based induction regimen, continuation maintenance with pemetrexed and bevacizumab seems to be at least equivalent to the ECOG 4599 regimen, albeit far more expensive. The PRONOUNCE trial, a phase III randomized study, attempted to answer this question about the importance 
Optimal Treatment Strategies for Advanced NSCLC

of bevacizumab through comparing carboplatin and pemetrexed followed by pemetrexed maintenance versus carboplatin, paclitaxel, and bevacizumab followed by bevacizumab maintenance. ${ }^{27}$ Unfortunately, the primary end point was PFS without grade 4 toxicity (G4PFS). Inferences about PFS and OS are difficult to make. The study showed no difference in the regimens for the primary end point of G4PFS.

Another ongoing phase III study, ECOG 5508, will help answer this question through evaluating the efficacy of 3 maintenance arms using bevacizumab, pemetrexed, or both after induction with the ECOG 4599 regimen. This will be the first maintenance trial to use bevacizumab in the induction phase and allow for its discontinuation in one of the randomized maintenance arms. To date, no randomized trials of carboplatin and pemetrexed with or without bevacizumab have been conducted. Finally, each of these regimens has a significantly different toxicity profile, and cost, which should also be considered when making treatment decisions.

\section{Choosing the Optimal First-Line Treatment}

Many factors must be considered when choosing the optimal first-line treatment for patients with advanced NS-NSCLC with absence of EGFR mutations or ALK rearrangements. Evaluation of a patient's PS and comorbidities may be useful to eliminate certain drug options because of their toxicity profiles. Ultimately, identifying a regimen that balances least toxicity and maximal efficacy will be the optimal first-line treatment for any individual patient. Two key differentiating factors are whether a patient has a poor PS and whether the patient is eligible for bevacizumab. An algorithm that may help navigate the most important decisions when determining optimal treatment for a patient with advanced NSCLC is shown in Figure 1.

\section{Performance Status}

Historically, patients with impaired PS, particularly those with significant comorbidities, were considered for palliative care referral, palliative radiation when applicable, and hospice enrollment, because the median OS for these patients is much less than 6 months. ${ }^{28}$ In the ECOG 1594 trial, for example, the initial enrollment criteria included PS 2, with a planned stratification for those with PS
0 to 1 versus those with PS 2. After enrolling 66 patients with PS 2, a significantly higher rate of adverse events was noted and the protocol was amended to exclude this patient population. ${ }^{6}$ Many subsequent clinical trials have only included patients with a PS 0 or 1 . AVAPERL allowed the enrollment of patients with PS 2, but only 10 patients in this category were enrolled. Newer data may contradict this practice of excluding patients with PS 2, perhaps because of better tolerability of newer agents, such as pemetrexed, compared with those used in the ECOG 1594 trial. Results presented at the 2012 ASCO Annual Meeting of a phase III study comparing single-agent pemetrexed versus standard carboplatin and pemetrexed chemotherapy in patients with PS 2 showed that patients receiving the combination chemotherapy had improved OS (9.1 vs 5.6 months; HR, 0.57; 95\% CI, 0.41-0.79; $P=.001) .{ }^{29}$ Combination therapy was well tolerated in this patient population, with low rates of anemia, neutropenia, and treatmentrelated mortality. Similar results from a phase II study were reported at the 2013 ASCO Annual Meeting, favoring cisplatin and gemcitabine over gemcitabine alone in patients with PS $2 .{ }^{30}$ Many of the trials discussed were conducted when EGFR and ALK testing was not standard, and therefore may have included patients who would have benefited from targeted first-line therapy. The interpretation of these results in terms of PS may therefore be difficult to interpret today, when most patients treated with cytotoxic first-line therapy are EGFR and ALK wild-type.

\section{Bevacizumab Eligibility}

After PS, the next major decision is whether to include bevacizumab in the induction regimen. $\mathrm{Pa}$ tients with squamous histology were identified to have a high rate of fatal pulmonary hemorrhage in a phase II study of bevacizumab, and all subsequent studies have excluded patients with squamous histology. ${ }^{9}$ The labeling of bevacizumab carries a black box warning for an increased risk of severe or fatal bleeding related to hemoptysis, gastrointestinal bleeding, central nervous system (CNS) bleeding, epistaxis, or vaginal bleeding, which is up to 5 times higher than this risk in patients receiving chemotherapy without bevacizumab. ${ }^{31} \mathrm{~A}$ meta-analysis of 15 prospective randomized trials using bevacizumab in many different malignancies, including NSCLC, showed a 33\% increase in risk for venous thromboembolism. ${ }^{32}$ The risk of arterial thrombotic events, such as cerebral 


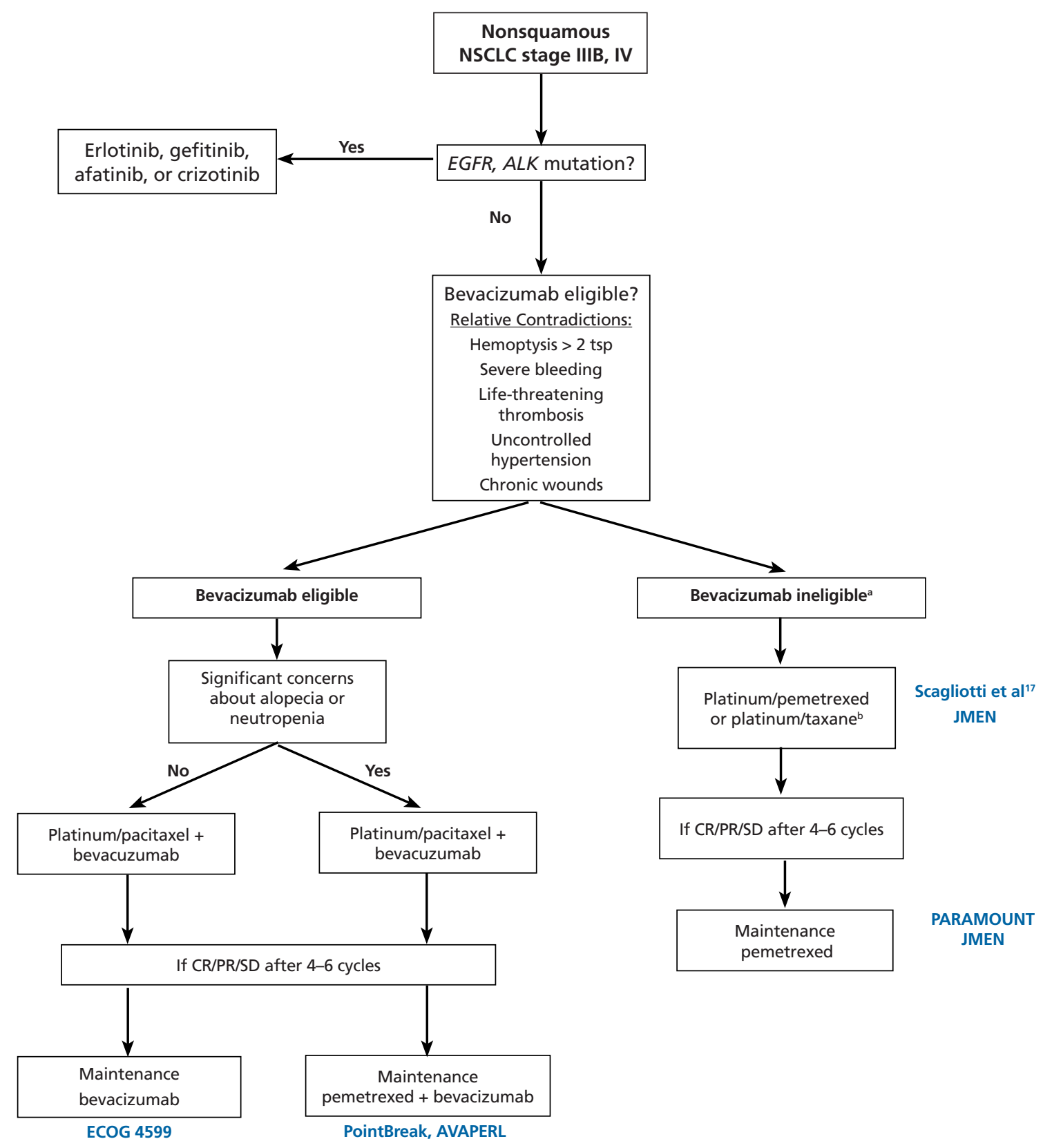

Figure 1 An algorithm for choosing first-line and maintenance therapy for patients with nonsquamous non-small cell lung cancer (NSCLC). Clinical trials supporting treatment options are listed.

Abbreviations: ALK, anaplastic lymphoma kinase; CR, complete response; EGFR, epidermal growth factor receptor; PR, partial response; SD, stable disease. aPatients who are eligible for bevacizumab may also be treated in this pathway because a nonbevacizumab regimen containing pemetrexed has never been compared head-to-head with one with bevacizumab. Other strategies, such as switch maintenance to pemetrexed, could also be considered. bOther agents could be considered, such as paclitaxel or docetaxel, which were used in the JMEN study. Gemcitabine should not be used in a nonsquamous population based on results of the trial by Scagliotti et al. ${ }^{17}$

and myocardial infarctions, is also higher in patients receiving bevacizumab versus other chemotherapy ( $2.6 \%$ vs $0.8 \%$, respectively, for grade 3 or 4 events). ${ }^{31}$ This risk was higher in patients with prior arterial thromboembolism or age older than 65 years. Lastly, grade 3 or 4 hypertension occurred in up to $18 \%$ of patients treated with bevacizumab. Recognizing many of these potential adverse effects, ECOG 4599 and subsequent studies using bevacizumab excluded patients with bleeding or coagulation disorders, use 
Optimal Treatment Strategies for Advanced NSCLC

of anticoagulants or higher doses of antiplatelet agents, or hemoptysis. ECOG 4599 also excluded patients with brain metastases, but subsequent trials, such as PointBreak, allowed stable, treated brain metastases and reported low rates of CNS bleeding for patients enrolled on the trial. Two phase II trials have demonstrated the safety of bevacizumab for patients with treated ${ }^{33}$ or asymptomatic untreated brain metastases. ${ }^{34}$ In consideration of the potential adverse events, first-line treatment of NSCLC, regardless of histology, should not include bevacizum$\mathrm{ab}$ for patients with recent history of hemoptysis of more than one-half teaspoon, severe bleeding event, arterial thrombosis, or poorly controlled hypertension despite medical management. Other reasons for omitting bevacizumab could include problems with chronic wound healing or recent surgery. Patients with stable, treated brain metastases can be eligible for treatment with bevacizumab.

Based on a meta-analysis of 4 trials, bevacizumab results in a $4 \%$ improvement in 1 -year survival (51-55\%). ${ }^{15}$ The degree of benefit in elderly patients ( $\geq 65$ years) is more debatable. In the original ECOG 4599 study, subgroup analysis showed that the benefit seemed to be limited to patients younger than 65 years (HR for age $\geq 65$ years: 0.89 ; 95\% CI, 0.70-1.14). ${ }^{10}$ In AVAiL, no difference was seen between the age groups, but fewer elderly patients were enrolled ( $31 \%$ vs $43 \%$ in ECOG 4599). ${ }^{11}$ A large retrospective analysis of the elderly population using Medicare claims in the SEER database was conducted for patients with NSCLC treated with or without bevacizumab between 2002 and 2007. ${ }^{35}$ Analysis of 4168 patients showed no statistically significant benefit of adding bevacizumab to carboplatin and paclitaxel in elderly patients. The median OS for patients receiving bevacizumab was 9.7 months versus 8.9 and 8.0 months in the 2 eras (HR, 1.01; 95\% CI, 0.88-1.15 and HR, 0.94; 95\% CI, 0.83-1.06, respectively) for patients receiving carboplatin and paclitaxel alone. However, other data presented at the 2012 ASCO Annual Meeting from N0821, a prospective phase II study in elderly patients receiving carboplatin, pemetrexed, and bevacizumab, suggests that elderly patients may benefit from a bevacizumab-containing regimen. In this single-arm study, patients older than 70 years had an impressive median PFS of 7.1 months. ${ }^{36}$ These data conflict with a retrospective analysis of ECOG 4599 that showed no benefit to bevacizumab in patients older than 70 years. $^{37}$

In summary, the data from these studies suggest that the additional benefit of bevacizumab in patients older than 70 years is less definitive. Age is often associated with other factors that may influence risk for bevacizumab use. Bevacizumab use as a standard treatment in elderly patients should be cautioned, and the decision to include bevacizumab must be made on an individual basis.

\section{Choosing the Platinum Partner}

Many active agents can be paired with cisplatin or carboplatin for the first-line treatment of NSCLC. ECOG 1594 showed that paclitaxel, docetaxel, and gemcitabine were equivalent, but that a combination of carboplatin with paclitaxel was easier to administer and had fewer adverse effects. ${ }^{6}$ Scagliotti et $\mathrm{al}^{17}$ showed that pemetrexed was highly active in NS-NSCLC and was superior to gemcitabine. For these reasons, pemetrexed and paclitaxel have been the 2 most commonly used platinum partners in most clinical trials and have only been compared head-to-head indirectly in the PointBreak maintenance trial. Choosing the appropriate platinum partner and maintenance strategy depends on whether bevacizumab will be included in the regimen.

\section{Bevacizumab Ineligible}

Bevacizumab may be contraindicated for various reasons, such as advanced age, hemoptysis, and other comorbidities that preclude its use. The PARAMOUNT and JMEN trials both showed improvement in OS with maintenance pemetrexed. . $^{21,24}$ PARAMOUNT used pemetrexed in the induction regimen and JMEN allowed paclitaxel, docetaxel, or gemcitabine during induction. Gemcitabine and erlotinib maintenance have also been shown to improve PFS after a gemcitabine-containing induction regimen, but failed to reach OS efficacy. ${ }^{20}$ Given that pemetrexed has been shown to be superior to gemcitabine for NS-NSCLC, paclitaxel or pemetrexed followed by pemetrexed maintenance are commonly used approaches. These 2 approaches without bevacizumab have never been compared head-to-head.

\section{Bevacizumab Eligible}

For patients without any contraindication to bevacizumab, the decision process is not entirely different from that for patients who are not eligible. Three main strategies have been studied: paclitaxel in ECOG 
4599, pemetrexed in PointBreak and AVAPERL, and gemcitabine in AVAiL. This article focuses on paclitaxel and pemetrexed, because gemcitabine is likely inferior for NS-NSCLC. The best data available comparing the ECOG 4599 and PointBreak regimens comes from PointBreak, in which both regimens and their respective maintenance strategies were shown to be equivalent in terms of OS. The difference of 2 weeks in PFS is not likely clinically significant. Other smaller studies have paired bevacizumab with pemetrexed or paclitaxel. ${ }^{19}$ The previously mentioned phase II N0821 study showed promising PFS results in an elderly population when combining pemetrexed with bevacizumab. ${ }^{36}$ These conflicting results do not suggest any clear advantage to either paclitaxel or pemetrexed as partners with bevacizumab. However, what may be clinically significant for some patients is the difference in toxicities, which were clearly seen in PointBreak, wherein the 2 combinations were directly compared. For patients with significant concerns regarding alopecia or peripheral neuropathy, pemetrexed may be favorable. For patients at risk for infectious complications, such as those who are immunocompromised or have autoimmune disorders, pemetrexed seems to have a lower incidence of neutropenia and febrile neutropenia. For most other patients, including those with concerns for thrombocytopenia, paclitaxel-containing regimens remain the standard of care for bevacizumabeligible patients. For those receiving pemetrexed and bevacizumab, the data from AVAPERL showing an improved PFS support the continuation of both pemetrexed and bevacizumab in the maintenance setting. This strategy was also shown to be equivalent in the PointBreak trial. Whether pemetrexed maintenance without bevacizumab in this instance would be equivalent is unclear. Forthcoming data from ECOG 5508 may help answer this question.

\section{Conclusions}

In the past decade, many significant clinical trials have advanced the understanding of lung cancer treatment, new drugs have become available, and the complexity of determining the optimal treatment strategy for patients with advanced NSCLC has increased significantly. Important factors to consider include histology, mutation status, PS, age, comorbidities and other factors that preclude the use of bevacizumab, and the desire to avoid alopecia and neutropenic fever. For most patients with NS-NSCLC, optimal first-line treatment is a combination of a platinum drug with pemetrexed alone, pemetrexed and bevacizumab, or paclitaxel and bevacizumab. Maintenance strategies for those without disease progression after each regimen include pemetrexed, pemetrexed and bevacizumab, or bevacizumab alone, respectively.

\section{References}

1. SEER Cancer Statistics Factsheets: Lung and Bronchus Cancer National Cancer Institute. Surveillance, Epidemiology, and End Results Program. Available at: http://seer.cancer.gov/statfacts/ html/lungb.html. Accessed April 21, 2014.

2. Ettinger DS, Wood DE, Akerley W, et al. NCCN Clinical Practice Guidelines in Oncology: Non-Small Cell Lung Cancer. Version 3, 2014. Available at: NCCN.org. Accessed April 21, 2014.

3. Non-small Cell Lung Cancer Collaborative Group. Chemotherapy in non-small cell lung cancer: a meta-analysis using updated data on individual patients from 52 randomised clinical trials. BMJ 1995;311:899-909.

4. Cullen MH, Billingham LJ, Woodroffe CM, et al. Mitomycin, ifosfamide, and cisplatin in unresectable non-small-cell lung cancer: effects on survival and quality of life. J Clin Oncol 1999;17:3188-3194.

5. Spiro SG, Rudd RM, Souhami RL, et al. Chemotherapy versus supportive care in advanced non-small cell lung cancer: improved survival without detriment to quality of life. Thorax 2004;59:828-836.

6. Schiller JH, Harrington D, Belani CP, et al. Comparison of four chemotherapy regimens for advanced non-small-cell lung cancer. N Engl J Med 2002;346:92-98.

7. Hotta K, Matsuo K, Ueoka H, et al. Meta-analysis of randomized clinical trials comparing cisplatin to carboplatin in patients with advanced non-small-cell lung cancer. J Clin Oncol 2004;22:3852-3859.

8. Seto T, Higashiyama M, Funai $H$, et al. Prognostic value of expression of vascular endothelial growth factor and its flt-1 and KDR receptors in stage I non-small-cell lung cancer. Lung Cancer 2006;53:91-96.

9. Johnson DH, Fehrenbacher L, Novotny WF, et al. Randomized phase II trial comparing bevacizumab plus carboplatin and paclitaxel with carboplatin and paclitaxel alone in previously untreated locally advanced or metastatic non-small-cell lung cancer. J Clin Oncol 2004;22:2184-2191.

10. Sandler A, Gray R, Perry MC, et al. Paclitaxel-carboplatin alone or with bevacizumab for non-small-cell lung cancer. N Engl J Med 2006;355:2542-2550.

11. Reck M, von Pawel J, Zatloukal P, et al. Phase III trial of cisplatin plus gemcitabine with either placebo or bevacizumab as first-line therapy for nonsquamous non-small-cell lung cancer: AVAil. J Clin Oncol 2009;27:1227-1234.

12. Reck M, von Pawel J, Zatloukal $P$, et al. Overall survival with cisplatin-gemcitabine and bevacizumab or placebo as first-line therapy for nonsquamous non-small-cell lung cancer: results from 
Optimal Treatment Strategies for Advanced NSCLC

a randomised phase III trial (AVAiL). Ann Oncol 2010;21:18041809.

13. Yang K, Wang YJ, Chen XR, et al. Effectiveness and safety of bevacizumab for unresectable non-small-cell lung cancer: a metaanalysis. Clin Drug Investig 2010;30:229-241.

14. Botrel TE, Clark O, Clark L, et al. Efficacy of bevacizumab (Bev) plus chemotherapy (CT) compared to CT alone in previously untreated locally advanced or metastatic non-small cell lung cancer (NSCLC): systematic review and meta-analysis. Lung Cancer 2011;74:89-97.

15. Soria JC, Mauguen A, Reck M, et al. Systematic review and metaanalysis of randomised, phase II/III trials adding bevacizumab to platinum-based chemotherapy as first-line treatment in patients with advanced non-small-cell lung cancer. Ann Oncol 2013;24:20-30.

16. Hanna N, Shepherd FA, Fossella FV, et al. Randomized phase III trial of pemetrexed versus docetaxel in patients with non-smallcell lung cancer previously treated with chemotherapy. J Clin Oncol 2004;22:1589-1597.

17. Scagliotti GV, Parikh P, von Pawel J, et al. Phase III study comparing cisplatin plus gemcitabine with cisplatin plus pemetrexed in chemotherapy-naive patients with advanced-stage non-small-cell lung cancer. J Clin Oncol 2008;26:3543-3551.

18. Barlesi F, Scherpereel A, Rittmeyer A, et al. Randomized phase III trial of maintenance bevacizumab with or without pemetrexed after first-line induction with bevacizumab, cisplatin, and pemetrexed in advanced nonsquamous non-small-cell lung cancer: AVAPERL (MO22089). J Clin Oncol 2013;31:30043011.

19. Patel JD, Socinski MA, Garon EB, et al. A randomized, openlabel, phase 3 , superiority study of pemetrexed $(\mathrm{Pem})+$ carboplatin $(\mathrm{Cb})+$ bevacizumab $(\mathrm{B})$ followed by maintenance pem $+\mathrm{B}$ versus paclitaxel $(\mathrm{Pac})+\mathrm{Cb}+\mathrm{B}$ followed by maintenance $\mathrm{B}$ in patients (pts) with stage IIIB or IV non-squamous non-small cell lung cancer (NS-NSCLC). J Thoracic Oncol 2012;7:S336-338.

20. Perol M, Chouaid C, Perol D, et al. Randomized, phase III study of gemcitabine or erlotinib maintenance therapy versus observation, with predefined second-line treatment, after cisplatin-gemcitabine induction chemotherapy in advanced nonsmall-cell lung cancer. J Clin Oncol 2012;30:3516-3524.

21. Paz-Ares LG, de Marinis F, Dediu M, et al. PARAMOUNT: final overall survival results of the phase III study of maintenance pemetrexed versus placebo immediately after induction treatment with pemetrexed plus cisplatin for advanced nonsquamous nonsmall-cell lung cancer [published online ahead of print July 8, 2013]. J Clin Oncol. doi:10.1200/JCO.2012.47.1102.

22. Patel JD, Hensing TA, Rademaker A, et al. Phase II study of pemetrexed and carboplatin plus bevacizumab with maintenance pemetrexed and bevacizumab as first-line therapy for nonsquamous non-small-cell lung cancer. J Clin Oncol 2009;27:3284-3289.

23. Paz-Ares L, de Marinis F, Dediu M, et al. Maintenance therapy with pemetrexed plus best supportive care versus placebo plus best supportive care after induction therapy with pemetrexed plus cisplatin for advanced non-squamous non-small-cell lung cancer (PARAMOUNT): a double-blind, phase 3, randomised controlled trial. Lancet Oncol 2012;13:247-255.

24. Ciuleanu T, Brodowicz T, Zielinski C, et al. Maintenance pemetrexed plus best supportive care versus placebo plus best supportive care for non-small-cell lung cancer: a randomised, double-blind, phase 3 study. Lancet 2009;374:1432-1440.

25. Rittmeyer A, Scherpereel A, Gorbunova VA, et al. Effect of maintenance bevacizumab (Bev) plus pemetrexed (Pem) after first-line cisplatin/Pem/Bev in advanced nonsquamous non-small cell lung cancer (nsNSCLC) on overall survival (OS) of patients (pts) on the AVAPERL (MO22089) phase III randomized trial [abstract]. J Clin Oncol 2013;31(Suppl):Abstract 8014.

26. Cappuzzo F, Ciuleanu T, Stelmakh L, et al. Erlotinib as maintenance treatment in advanced non-small-cell lung cancer: a multicentre, randomised, placebo-controlled phase 3 study. Lancet Oncol 2010;11:521-529.

27. Zinner R, Ross HJ, Weaver R, et al. Randomized, openlabel, phase III study of pemetrexed plus carboplatin (PemC) followed by maintenance pemetrexed versus paclitaxel/ carboplatin/bevacizumab (PCB) followed by maintenance bevacizumab in patients with advanced nonsquamous (NS) non-small cell lung cancer (NSCLC) [abstract]. J Clin Oncol 2013;31(Suppl):Abstract LBA8003.

28. Lee SM, Khan I, Upadhyay S, et al. First-line erlotinib in patients with advanced non-small-cell lung cancer unsuitable for chemotherapy (TOPICAL): a double-blind, placebo-controlled, phase 3 trial. Lancet Oncol 2012;13:1161-1170.

29. Lilenbaum R, Zukin M, Pereira JR, et al. A randomized phase III trial of single-agent pemetrexed $(\mathrm{P})$ versus carboplatin and pemetrexed $(\mathrm{CP})$ in patients with advanced non-small cell lung cancer (NSCLC) and performance status (PS) of 2 [abstract]. J Clin Oncol 2012;30(Suppl):Abstract 7506.

30. Morabito A, Gebbia V, Cinieri S, et al. Randomized phase III trial of gemcitabine and cisplatin versus gemcitabine alone in patients with advanced non-small cell lung cancer (NSCLC) and a performance status (PS) 2: CAPPA-2 study [abstract]. J Clin Oncol 2013;31(Suppl):Abstract 8066.

31. Avastin [package insert]. South San Francisco, CA: Genentech, Inc; 2013.

32. Nalluri SR, Chu D, Keresztes R, et al. Risk of venous thromboembolism with the angiogenesis inhibitor bevacizumab in cancer patients: a meta-analysis. JAMA 2008;300:2277-2285.

33. Socinski MA, Langer CJ, Huang JE, et al. Safety of bevacizumab in patients with non-small-cell lung cancer and brain metastases. J Clin Oncol 2009;27:5255-5261.

34. Besse B, Le Moulec S, Senellart H, et al. Phase II study of bevacizumab in combination with first-line chemotherapy or second-line erlotinib in non-squamous NSCLC patients with asymptomatic untreated brain metastases (ML21823). Ann Oncol 2012;23:ix426.

35. Zhu J, Sharma DB, Gray SW, et al. Carboplatin and paclitaxel with vs without bevacizumab in older patients with advanced non-small cell lung cancer. JAMA 2012;307:1593-1601.

36. Dy GK, Molina JR, Qi Y, et al. N0821: a phase II first-line study of a combination of pemetrexed (P), carboplatin (C), and bevacizumab (B) in elderly patients with good performance status (PS < 2) [abstract]. J Clin Oncol 2012;30(Suppl 1):Abstract 7555.

37. Ramalingam SS, Dahlberg SE, Langer CJ, et al. Outcomes for elderly, advanced-stage non small-cell lung cancer patients treated with bevacizumab in combination with carboplatin and paclitaxel: analysis of Eastern Cooperative Oncology Group Trial 4599. J Clin Oncol 2008;26:60-65. 